\title{
A Modern High-Precision Calculation of Deep Underground Cosmic Ray Muons
}

\author{
William Woodley, ${ }^{a, *}$ Anatoli Fedynitch ${ }^{b}$ and Marie-Cécile Piro ${ }^{a}$ \\ ${ }^{a}$ University of Alberta, Department of Physics \\ 4-181 CCIS, 116 St. and 85 Ave., Edmonton, AB T6G 2R3, Canada \\ ${ }^{b}$ Institute for Cosmic Ray Research, University of Tokyo, \\ 5-1-5 Kashiwa-no-ha, Kashiwa, Chiba 277-8582, Japan \\ E-mail: wwoodley@ualberta.ca, afedyni@icrr.u-tokyo.ac.jp, \\ mariecci@ualberta.ca
}

We present a new efficient calculation to propagate cosmic ray muons from the surface of the Earth to deep underground laboratories, allowing us to look at the physics and performance of various models of high-energy cosmic rays. The evolution of cosmic rays in the Earth's atmosphere is computed with MCEq (Matrix Cascade Equation), taking into account different combinations of primary and hadronic interaction models in order to calculate the muon flux at the surface. The latter serves as an input for the Monte Carlo code PROPOSAL (Propagator with Optimal Precision and Optimised Speed for All Leptons) to propagate the muons through the rock. A forward prediction for underground muon spectra at different slant depths, including the muon survival probabilities and underground energy spectra, is calculated with very high precision. The reliability of this state-of-the-art calculation was achieved by comparing the results obtained for the vertical muon intensity and total muon flux with the measured data at various underground sites with both flat overburdens and mountains. The implications of the results as well as the seasonal variation of the muon flux will also be discussed.

$37^{\text {th }}$ International Cosmic Ray Conference (ICRC 2021)

July 12 th - 23rd, 2021

Online - Berlin, Germany

\footnotetext{
*Presenter
} 


\section{Introduction}

The study of cosmic ray muons in detectors deep underground dates back many decades. The latest comprehensive analyses of underground measurements were performed 20 years ago by Klimushin, Bugaev, and Sokalski [1], and 15 years ago by Mei and Hime (MH) [2].

For practical applications, the muon intensity at a certain depth underground is often calculated using a parametric model, termed a Depth-Intensity Relation (DIR). Groom, et al. [3] give the following DIR for depth ranges between 1 and 10 km.w.e.:

$$
I(h)=I_{1} e^{\left(-h / \lambda_{1}\right)}+I_{2} e^{\left(-h / \lambda_{2}\right)},
$$

where $h$ is the vertical depth of the lab, and $I_{1}, I_{2}, \lambda_{1}$, and $\lambda_{2}$ are determined by fitting to experimental data. One issue with this method is that such phenomenological fits may contain bias induced by systematic uncertainties of the data. Alternatively, theoretical calculations, like those presented in [1], avoid this issue, but often lack a rigorous treatment of theoretical uncertainties.

Furthermore, muon intensities are often given in the literature in terms of vertical-equivalent intensities, calculated by multiplying the data by $\cos (\theta)$, where $\theta$ is the zenith angle. However, the muon flux at the surface only approximately scales with $1 / \cos (\theta)$, and the deviation between the vertical-equivalent intensity and the true vertical intensity (that for $\theta=0$ ) becomes significantly large as $\theta$ increases.

The motivation behind this work is to perform a new state-of-the-art calculation using two modern, open source, high-precision codes for the transport of cosmic rays through the atmosphere to deep underground. The aim is to achieve estimates of the underground muon flux, including its uncertainties, and to trigger more careful thought towards what the vertical-equivalent muon intensity is. Moreover, our results show that important constraints for the physics of hadronic interactions and cosmic ray fluxes at multi- $\mathrm{TeV}$ projectile energies can be obtained from highprecision underground muon data. A precise knowledge of muon-induced background processes is relevant for dark matter and neutrino detectors.

\section{Computational Method}

The calculation of the underground muon fluxes is divided into two parts: the first covers the journey of the muon from the atmosphere to the surface of the Earth, and is done using MCEq [4], and the second covers its journey from the surface of the Earth to the underground lab, and is done using PROPOSAL [5].

MCEq is a one-dimensional fast cascade equation solver for the muon flux at the surface, $\Phi^{s}$, as a function of muon energy at the surface, $E^{s}$, and zenith angle, $\theta$. The code takes into account the primary cosmic ray spectrum, the atmosphere, and the angular distributions. It can be used with various models for the primary cosmic ray flux and the hadronic interactions. The hadronic interaction models used for the results presented here are DDM, a recently-developed data-driven interaction model [6], and SiBYLL-2.3D, an event generator model [4]. The cosmic ray flux model is the Global Spline Fit [7].

PROPOSAL is a full Monte Carlo program that simulates the transport of leptons through long ranges of matter quickly and with high precision. The code takes into account discrete and 


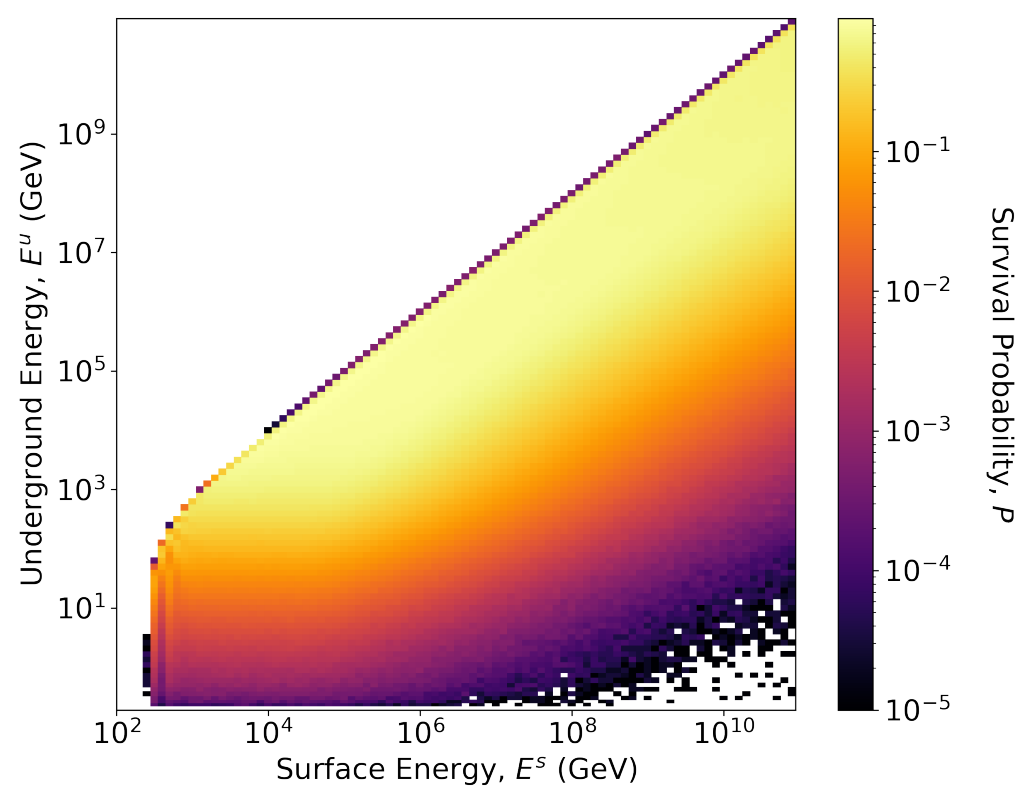

Figure 1: Surface-to-underground transfer matrix against surface energy and underground energy, for a depth of $h=1$ km.w.e., simulated with PROPOSAL for $10^{5}$ muons per surface energy-zenith angle bin.

continuous energy losses as well as the stopping and decaying of muons. It is used to calculate transfer matrices, as shown in Fig. 1, which give the probability that a muon of a given energy and zenith angle at the surface will survive the journey underground and end up with an energy $E^{u}$.

\subsection{Calculation of Underground Fluxes}

The underground muon flux, $\Phi^{u}$, is calculated by convolving the surface muon flux matrix from MCEq with the transfer matrix from PROPOSAL. For laboratories under flat earth, this is given by

$$
\Phi^{u}\left(E_{j}^{u}, X_{k}, \theta_{k}\right)=\sum_{i} \Phi^{s}\left(E_{i}^{s}, \theta_{k}\right) P\left(E_{i}^{s}, E_{j}^{u}, X_{k}\right)\left(\frac{\Delta E_{i}^{s}}{\Delta E_{j}^{u}}\right),
$$

where $X$ is slant depth, and $P$ is survival probability, which comes from the elements of the transfer matrix. The ratio of bin widths, $\left(\Delta E_{i}^{s} / \Delta E_{j}^{u}\right)$, is to account for the fact that muons starting in the same energy bin at the surface can end up in different energy bins underground, as muons do not always lose the same amount of energy while travelling through the rock.

For labs under mountains, the calculation is slightly more complicated; the amount of rock a muon has to travel through is dependent, in this case, on both the zenith angle and the azimuthal angle, and so the geometry of the mountain has to be taken into account in the calculations. To do this, topographic maps of the mountains in terms of $X(\theta, \phi)$ data are obtained from the labs (where $\phi$ is azimuthal angle), such as the one for Gran Sasso in Fig. 2 (left). The data is placed into bins of $X$ and $\cos (\theta)$, and is normalised, leading to a matrix of weights, $\hat{w}(X, \cos (\theta))$, shown in Fig. 2 (right). These weights are equivalent to the integration over the azimuthal angle for a given bin in $\theta$ and $X$, removing $\phi$ as a variable from the calculations, since, at the relevant energies, the surface 

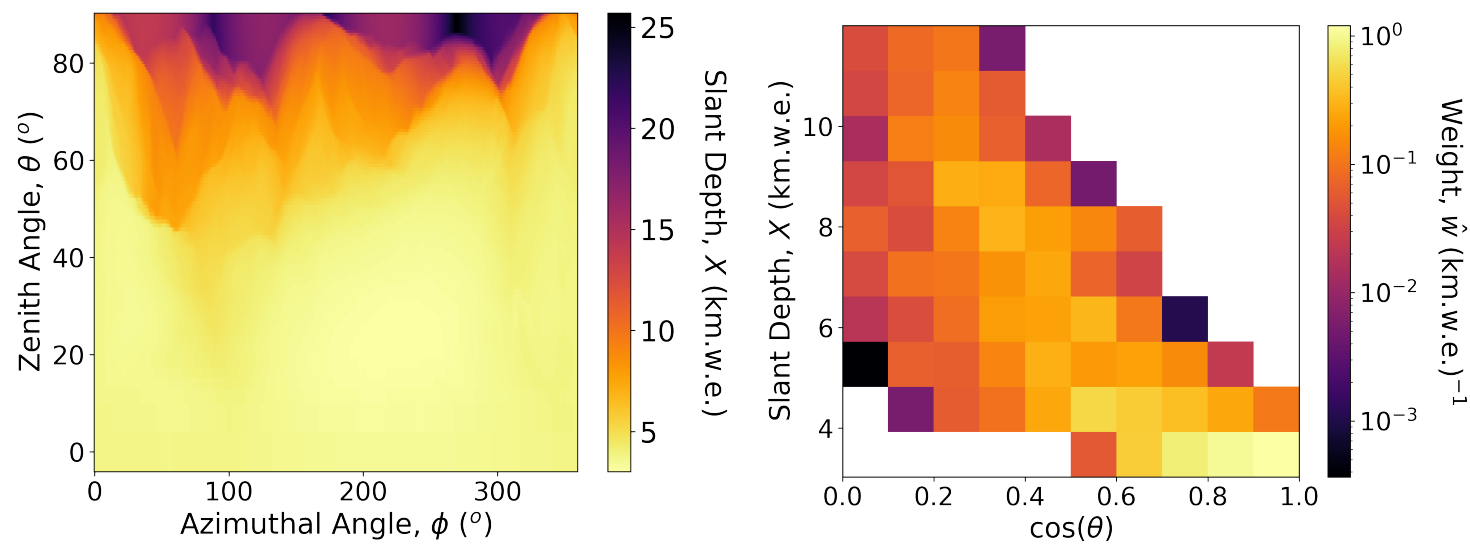

Figure 2: Left: The $X(\theta, \phi)$ data from the Gran Sasso topographic map, showing the profile of the mountain. Right: The normalised histogram in bins of $X$ and $\cos (\theta)$, giving the weight matrix.

fluxes are symmetric in $\phi$. The weights are then multiplied into the convolution in Eq. (2) to give the underground flux for labs under mountains.

\subsubsection{Underground Intensity}

The underground muon intensity, $I^{u}$, is obtained by integrating the underground flux calculated in Eq. (2) over underground energy:

$$
I^{u}(X, \theta)=\int_{E_{t h}}^{\infty} \Phi^{u}\left(E^{u}, X, \theta\right) \mathrm{d} E^{u},
$$

where $E_{t h}$ is the threshold energy that a muon needs in order to survive the journey underground to the lab, here set to zero, or a detection threshold of an experiment. In the literature, the verticalequivalent underground intensity, $I_{e q}^{u}$, is usually then calculated by multiplying the underground intensity from Eq. (3) by $\cos (\theta)$ :

$$
I_{e q}^{u}(X)=I^{u}(X, \theta) \cos (\theta) .
$$

However, because the surface flux does not perfectly scale with $1 / \cos (\theta)$, this approximation breaks down at larger zenith angles, in particular near-horizontal angles. Eq. (4) is insufficient for calculating intensities at anything but small zenith angles $<30^{\circ}$. Therefore, the true vertical underground intensity, $I_{t r}^{u}$, calculated using $\theta=0$,

$$
\begin{aligned}
I_{t r}^{u}(X) & =I^{u}(X, \theta=0) \\
& =\int_{E_{t h}}^{\infty} \sum_{i} \Phi^{s}\left(E_{i}^{s}, \theta_{k}=0\right) P\left(E_{i}^{s}, E_{j}^{u}, X_{k}\right)\left(\frac{\Delta E_{i}^{s}}{\Delta E_{j}^{u}}\right) \mathrm{d} E^{u},
\end{aligned}
$$

is more appropriate. While this aspect is widely discussed in the literature, some experiments still use the $1 / \cos (\theta)$ approximation for their results. In addition to this simplified view, it would be very valuable to see double differential measurements in $X$ and true zenith angle, $\theta$. 


\subsubsection{Total Underground Flux}

The total underground muon flux is calculated by integrating the intensity from Eq. (3) over all angles:

$$
\Phi_{\mathrm{tot}}^{u}(h)=2 \pi \int_{0}^{1} I^{u}\left(X=\frac{h}{\cos (\theta)}, \theta\right) \mathrm{d} \cos (\theta),
$$

where $h$ is the vertical depth underground, given by $h=X \cos (\theta)$, and the $2 \pi$ accounts for the integration over the azimuthal angle. For labs under mountains, the $2 \pi$ is not needed, because the dependence on the azimuthal angle is taken into account in the weights.

MH [2] proposed the definition of a "vertical equivalent depth" as the depth a lab would be at for the calculated flux if the lab were under a flat overburden. Using a fitted DIR, they calculate the total flux for labs under mountains, and then find the depths on the DIR that would correspond to the same flux under flat overburdens. We follow a similar approach: first, we compute our DIR by calculating the total muon flux for flat earth for a range of depths. The total flux for a lab under a mountain is then calculated using Eq. (6) (without the multiplication by $2 \pi$ ). The equivalent vertical depth of the lab is then the depth on our computed DIR that corresponds to that flux.

\section{Results}

\subsection{True Vertical Underground Intensity}

Our calculation of the true vertical underground muon intensity, using Eq. (5), for the two hadronic interaction models SiBYLL-2.3D and DDM is given in Fig. 3. There is excellent agreement between our results and the experimental data over the entire depth range. The new DDM [6] is better at describing the data at shallow slant depths, roughly corresponding to surface energies at several $100 \mathrm{GeV}$. SiBYLL-2.3D is better for deeper slant depths that sample surface energies from TeV to tens of TeV. Visually, the data aligns well with the MH fit; however, the slopes of individual data sets follow the trends of our calculations. It is beyond the scope of this contribution to discuss the impact of experimental systematic uncertainties. Remarkably, the error bars on some data points are smaller than those on the theory. This implies that, using this method, hadronic and cosmic ray uncertainties can be constrained.

\subsection{Total Underground Flux}

The total underground muon flux is calculated according to Eq. (6). As shown in Fig. 4, our result reproduces flat overburden labs (full circles) very well. For the case of labs located under mountains, the result of the calculation is the flux value. The vertical equivalent depth $h$ for these labs is found iteratively and is a second result. This value of $h$ does not correspond to the average depth that can be derived from the topographic map. The meaning behind $h$ is that it allows for a straightforward comparison of the expected muon-induced background rate for labs with very complex overburdens to those having a known vertical depth (such as SNOLAB). The MH fit is reproduced well, but the values in $h$ by construction lie on the curve (as in our case). Some significant differences occur for our calculations for Gran Sasso (green) and Kamioka (red; map from [11]). This is very likely related to different sources of topographic maps, since our flux values reproduce the data using the maps for Fréjus (from [12]) and Jinping. 

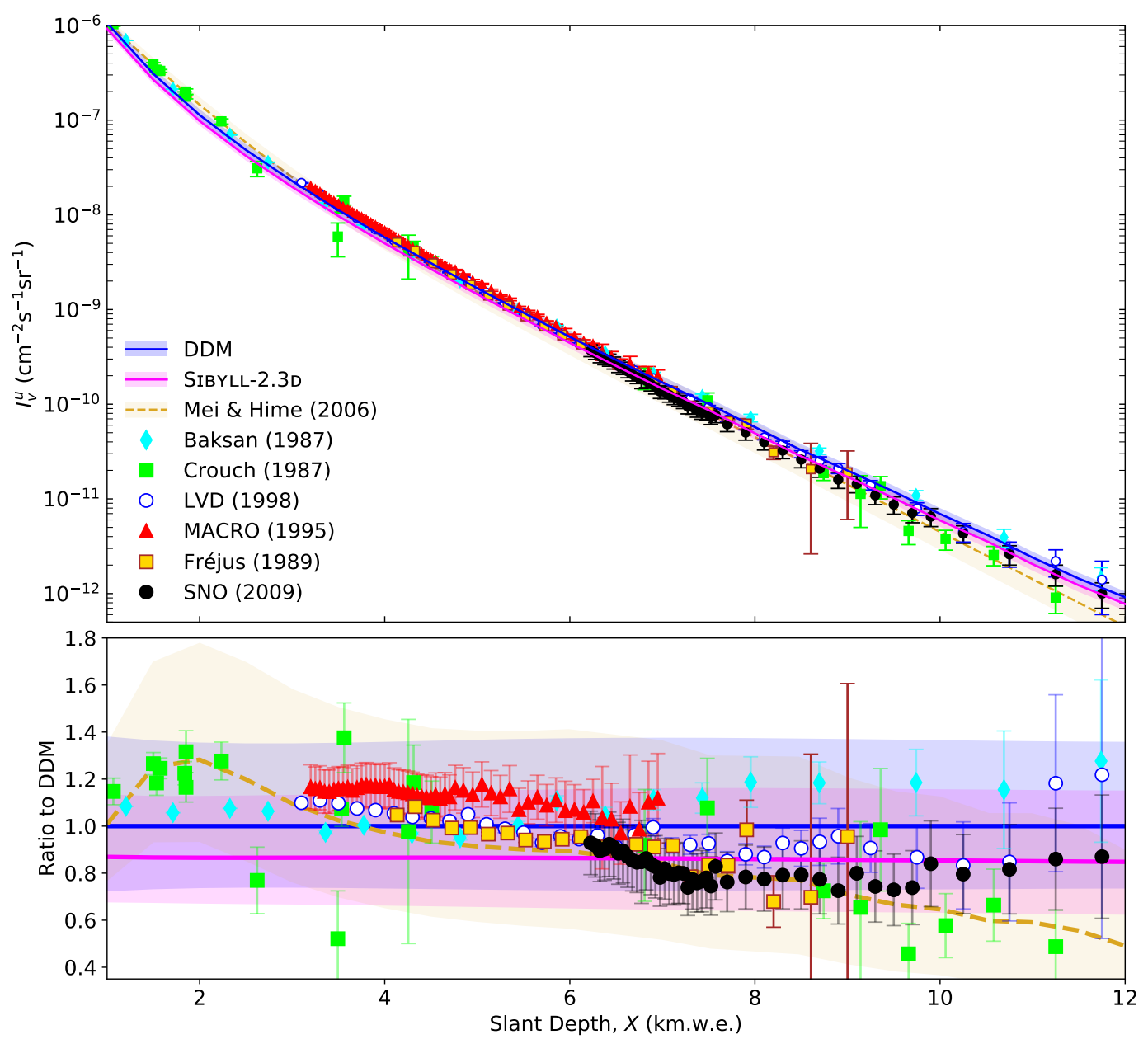

Figure 3: Top: True vertical underground muon intensity against slant depth for both SIBYLL-2.3D and DDM. Beyond 12 km.w.e., the intensity becomes dominated by neutrino-induced muons. Bottom: The ratio to DDM of the experimental data and the predictions made with SIBYLL-2.3D with uncertainties computed using the "Bartol" method [8], and with a parametric fit from MH with uncertainties derived from fitting Eq. (1) to data. All experimental data is referenced in [9]. Note that some error bars do not include systematic uncertainties.

\subsection{Seasonal Variations}

MCEq makes use the static atmospheric model NRLMSISE-00 [16] to simulate the variation of the muon flux across the seasons. The amplitude of the seasonal variations observed underground is affected by the depth of the lab, since deeper labs sample higher-energy muons. It is also affected by the location on Earth, as labs closer to the equator experience less change in their atmospheres across the seasons, and so the amplitudes of the variation, seen in Fig. 5, are less. The predictions using the simplified model coincide remarkably well with the measurements, despite not taking into account the actual atmospheric conditions that are subject to global warming or other extreme conditions. Overall, the seasonal variation contributes between $0.5 \%$ and $2 \%$ to the systematic errors in the results in Fig. 3 and Fig. 4, depending on the lab. 


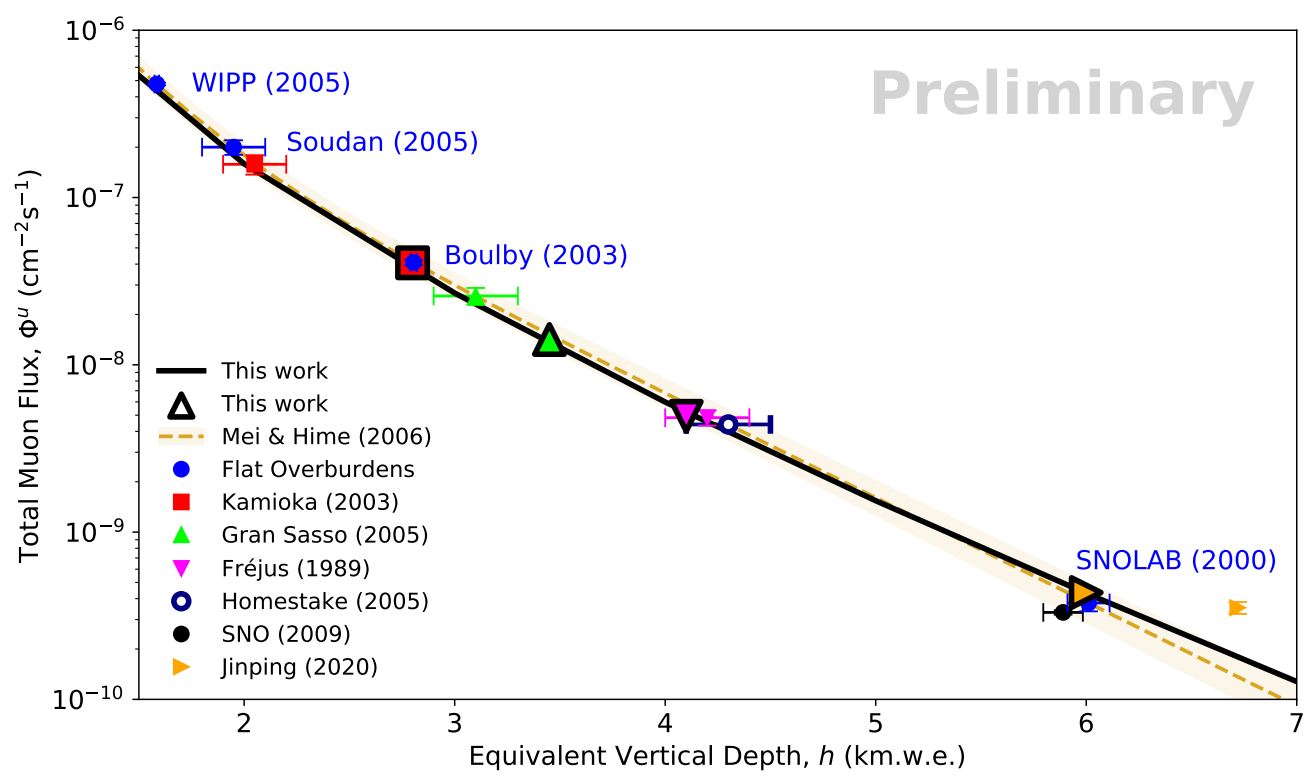

Figure 4: Total underground muon flux vs equivalent vertical depth. The black curve is our calculation for flat overburdens, and the points with black outlines are the results for labs under mountains, which have had $h$ adjusted to lie on the black curve. Experimental data is referenced in [2] and [10].

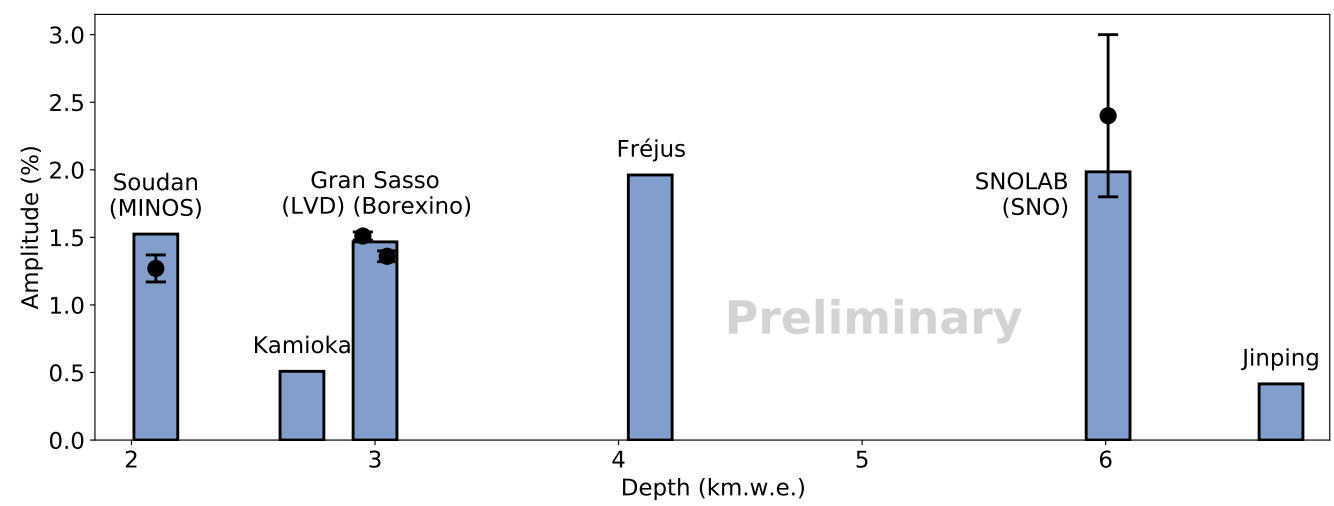

Figure 5: The amplitude of the seasonal variation of the underground flux seen by different labs, with experimental data points from [13-15].

\section{Conclusion}

By combining the modern codes MCEq and PROPOSAL, a program has been written to calculate cosmic ray muons fluxes deep underground. The program is fast, precise, and flexible, and the results match experimental data well. It can be used to calculate underground muon fluxes for dark matter and neutrino detectors in labs under either flat overburdens or mountains, and the seasonal variation of the flux can be simulated. Lastly, the results can be used to set constraints on uncertainties for hadronic interactions and cosmic ray fluxes. 


\section{Acknowledgements}

We acknowledge the help of Marco Selvi, who provided us with a topographic map of the Gran Sasso mountain from LVD data, as well as Shaomin Chen, who provided us with a topographic map of the Jinping mountain from JNE data. We acknowledge, as well, the help of Michel Zampaolo and Luigi Mosca for the data of the Fréjus detector and Shigetaka Moriyama for the map of Kamioka. AF acknowledges the support from the JSPS (KAKENHI 19F19750). MCP acknowledges the support from the McDonald Institute.

\section{References}

[1] S.I. Klimushin, E.V. Bugaev and I.A. Sokalski, Phys. Rev. D 64 (2001) 014016 [hep-ph/0012032].

[2] D. Mei and A. Hime, Phys. Rev. D 73 (2006) 053004 [astro-ph/0512125].

[3] G.L. Cassiday, J.W. Keuffel and J.A. Thompson, Phys. Rev. D 7 (1973) 2022.

[4] A. Fedynitch, F. Riehn, R. Engel, T.K. Gaisser and T. Stanev, Phys. Rev. D 100 (2019) 103018.

[5] J.-H. Koehne, K. Frantzen, M. Schmitz, T. Fuchs, W. Rhode, D. Chirkin et al., Computer Physics Communications 184 (2013) 2070.

[6] A. Fedynitch and M. Huber, PoS ICRC2021 (2021) 1076.

[7] H.P. Dembinski, R. Engel, A. Fedynitch, T. Gaisser, F. Riehn and T. Stanev, PoS ICRC2017 (2018) 533 [1711.11432].

[8] G.D. Barr, T.K. Gaisser, S. Robbins and T. Stanev, Phys. Rev. D 74 (2006) 094009 [astro-ph/0611266].

[9] Particle Data Group, PTEP 2020 (2020) 083C01.

[10] SNO Collaboration, Phys. Rev. D 80 (2009) 012001.

[11] KamLand Collaboration, Phys. Rev. C 81 (2010) 025807.

[12] Fréjus Collaboration, Phys. Rev. D 40 (1989) 2163.

[13] MinOS Collaboration, Phys. Rev. D 91 (2015) 112006.

[14] M. Agostini, K. Altenmüller, S. Appel, V. Atroshchenko, Z. Bagdasarian, D. Basilico et al., Journal of Cosmology and Astroparticle Physics 2019 (2019) 046.

[15] C. Kyba, Ph.D. thesis, University of Pennsylvania, 2006.

[16] J.M. Picone, A.E. Hedin, D.P. Drob and A.C. Aikin, Journal of Geophysical Research: Space Physics 107 (2002) SIA 15. 